\title{
Botulinum Toxin Injection in the Treatment of Postextubation Dysphagia: A Case Report
}

\author{
Byung Wook Kim, MD ${ }^{1}$, Hee-Ju Kim, MD ${ }^{1}$, Jung Keun Hyun, MD, PhD ${ }^{1,2,3}$, \\ Seo Young Kim, $\mathrm{MD}^{1}$, Tae Uk Kim, $\mathrm{MD}, \mathrm{PhD}^{1}$

\begin{abstract}
${ }^{1}$ Department of Rehabilitation Medicine, Dankook University College of Medicine, Cheonan;
\end{abstract} \\ ${ }^{2}$ Department of Nanobiomedical Science \& WCU Research Center, Dankook University, Cheonan; \\ ${ }^{3}$ Institute of Tissue Regeneration Engineering (ITREN), Dankook University, Cheonan, Korea
}

Prolonged intubation is known to bring on postextubation dysphagia (PED) in some patients. We have noted that there were some studies to investigate specific type and pattern of PED, which showed large variety of different swallowing abnormalities as mechanisms of PED that are multifactorial. There are several options of treatment in accordance with the management of these abnormalities. A botulinum toxin (BoT) injection into the upper esophageal sphincter (UES) can improve swallowing functions for patients with this disorder, by working to help the muscle relax. In this case, the conventional treatment was not effective in patients with PED, whereas the BoT injection made a great improvement for these patients. This study suggests that the UES pathology could be the main cause of PED.

Keywords Deglutition disorders, Endotracheal intubation, Botulinum toxin

\section{INTRODUCTION}

Postextubation dysphagia (PED) is defined as the difficulty in effectively and safely transferring food and liquid from the mouth to the stomach after a patient has experienced the procedure of extubation [1]. Though the term prolonged intubation varied from 8 hours to several months in previous literature [2], endotracheal intubation longer than 48 hours has been shown to considerably increase the risk for dysphagia with a reported prevalence of $34 \%-56 \%$ of these cases [3]. Patients with PED show a large variety of aspiration types and associated swallowing disorders, including noted upper esophageal sphincter (UES) dysfunction as seen in a reviewed videofluoroscopic swallowing study (VFSS) [4]. There are several treatments in PED: dietary texture modifications, postural changes, compensatory maneuvers, and interventions to improve swallowing functions such as therapeutic exercise, neuromuscular electrical stimulation, along with the use of an UES botulinum toxin (BoT)

Department of Rehabilitation Medicine, Dankook University College of Medicine, 201 Manghyang-ro, Dongnam-gu, Cheonan 31116, Korea. Tel: +8241-550-6641, Fax: +82-41-551-7062, E-mail: magnarbor@dankook.ac.kr

ORCID: Byung Wook Kim (http://orcid.org/0000-0002-9456-9294); Hee-Ju Kim (http://orcid.org/0000-0001-6877-5126); Jung Keun Hyun (http://orcid. org/0000-0001-9254-4424); Seo Young Kim (http://orcid.org/0000-0002-5351-8044); Tae Uk Kim (http://orcid.org/0000-0001-9904-8781).

(c) This is an open-access article distributed under the terms of the Creative Commons Attribution Non-Commercial License (http://creativecommons.org/ licenses/by-nc/4.0) which permits unrestricted noncommercial use, distribution, and reproduction in any medium, provided the original work is properly cited. Copyright $\odot 2018$ by Korean Academy of Rehabilitation Medicine 
injection. Several studies, according to a literature review, have been shown positive effects of BoT injection in patients with various types of dysphagia who have neurological disorders [5,6], but studies on BoT injection in PED derived from UES dysfunction without previous neurological disorders are lacking. In this study, we report a case of BoT injection in patients with PED who have no neurological disorders. This study was approved by the Institutional Review Board of Dankook University Hospital (No. 2017-08-001).

\section{CASE REPORT}

The patient is a 67 -year-old woman with no history of stroke, spinal cord injury, or other neurological disorders, who was subsequently admitted to an intensive care unit (ICU) due to dyspnea. The patient had been taking medicines to control hypertension, atrial fibrillation, and had a left hemithyroidectomy as diagnosed approximately 40 years ago. The patient was diagnosed with asthma approximately 3 years ago, and this diagnosed condition appeared to become worsened at this time. The patient is noted to have suffered from a cough, sputum and fever for 1 week. The results of the patient's chest X-ray showed a collapsed left lower lobe of lung. After rapid intubation, the patient was brought back to a stable condition status gradually over time. Despite the reduction of the fraction of inspired oxygen, the patient's saturation of peripheral oxygen was maintained above $95 \%$. At that time, the patient was transferred to general ward after extubation was performed. The duration of endotracheal tube placement for this patient was observed as taking 57 hours and 10 minutes.

In general ward, the patient complained of hoarseness and a sore throat. The patient presented with a paralyzed left vocal cord and bowed right vocal cord, which were found through the utilization of a laryngoscopy by an otolaryngologist, who therefore planned an injection laryngoplasty (IL) to improve these noted symptoms. After receiving the extubation, the patient also complained of reflex cough when she swallowed something, which suggested the incidence and presence of PED. The patient was examined with videofluoroscopic swallowing study (VFSS). The report showed the case of a profoundly decreased laryngeal elevation and epiglottic folding, incomplete laryngeal closure, inappropriate relaxation of UES, moderate residues in pyriform sinus and a massive aspiration (Fig. 1A). It was noted that the patient's rating as measured by the American Speech-LanguageHearing Association National Outcome Measurement System (ASHA NOMS) Swallowing Scale was rated at the number and level status of 1 . Consequently, the patient maintained a nasogastric tube, and had swallowing training including postural changes and compensatory ma-
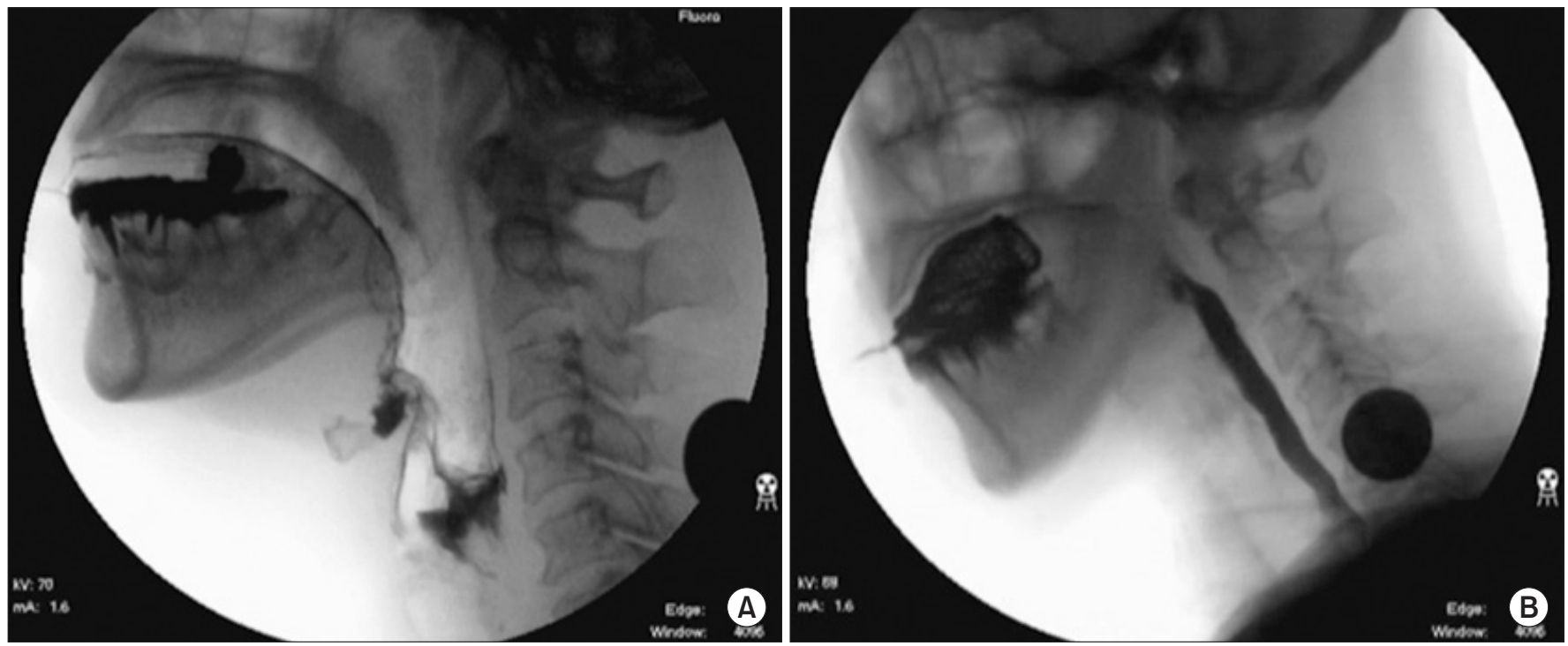

Fig. 1. (A) Before the botulinum toxin injection, inappropriate relaxation of upper esophageal sphincter and massive aspiration is shown. (B) After the botulinum toxin injection, laryngeal elevation and relaxation of upper esophageal sphincter were significantly enhanced. The patient adopted chin tuck posture. 
neuvers to assist with the management of this function. Additionally the patient received functional electrical stimulation (FES) for 4 weeks. In this case, the VFSS was examined every week after swallowing training and FES, but it did not improve for 1 month and a half afterwards.

For the attempt to utilize and apply alternative approaches, the patient was given a BoT injection for the management of the insufficient opening of the UES. There were a total of 20 units of BoT that were injected into the patient on two location sites of UES (Fig. 2). After receiving the BoT injection, it is noted that the patient's laryngeal elevation, epiglottic folding and relaxation of the UES were significantly enhanced. In short, it should be noted that neither the aspiration, nor the penetration was shown at that time (Fig. 1B). The ASHA NOMS was 5 at that time. The patient's symptoms were noted, suggesting that the PED was much improved. The IL was also performed at the same time for the management of hoarseness and sore throat. As a result, the patient was able to manage to therefore eat a soft diet, and tolerate drinking water with thickener as taken by mouth. The patient was eventually discharged without the use of a nasogastric tube.

After 2 months, the follow-up study regarding the patient was reviewed, and the patient was examined at the outpatient clinic. Contrary to the doctors' expectations of the patient's progress, it was noted that the results were aggravated at the time of review. In that case, a silent aspiration was observed when the patient drank fluid from

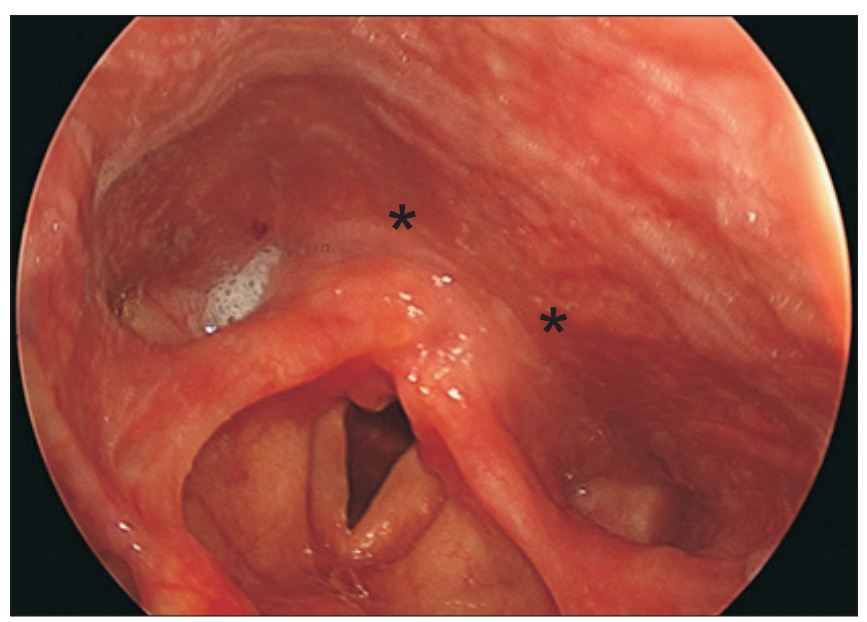

Fig. 2. The asterisks indicate the two injection sites of upper esophageal sphincter on laryngoscopy. a cup, which we surmise was due to an incomplete laryngeal closure, as noted with decreased laryngeal elevation and epiglottic folding. The relaxation of UES was also inappropriate.

\section{DISCUSSION}

We further note that the mechanism of PED is multifactorial, which includes mechanical causes, cognitive disturbances, and residual effects of narcotics and anxiolytic medications. The mechanical causes are noted to be directly related to the duration of intubation in the patient [1]. We bring attention to the issue of a prolonged intubation, which is known to cause mucosal inflammation, with loss of its architecture and can exacerbate a subsequent laryngeal injury. This procedure can also cause oropharyngeal muscle atrophy during intubation, diminished proprioception and laryngeal sensation [1]. This injury can cause the pathology of the UES, given that the main opening force of the UES is movement of the entire larynx upward and forward under the tongue base [7].

There were some studies that investigate the effects of BoT injection to patients with neurological disorders such as stroke, Parkinson's disease, multiple sclerosis, multiple system atrophy, systemic lupus erythematosus, etc. The success rate of the use of a BoT injection in these cases was approximately $50 \%[5,6]$. The aforementioned neurological disorders interfere with sequential and coordinated movements of the facial, cervical, oral, pharyngeal, laryngeal and esophageal muscles, and therefore as a result it leads to absolute or relative hypertonicity of UES. It is noted to this end, that the BoT injection reduces the tonic UES contraction, and for this reason, these patients can benefit from the BoT injection. Conversely, it is difficult to manage or focus on the hypertonicity of UES from the beginning in patients with PED who have no neurological disorders. The normally functioning UES maintains esophageal closure at rest, and briefly relaxes to allow for bolus transit. The other two opening forces of UES are from the intrabolus pressure from above and the intrinsic relaxation of UES [7]. The intrabolus pressure from above can be reduced in the patients with PED, especially in the case whether the patient has neurological disorders or not, and the BoT injection can help the bolus to pass through the UES more easily by making tone 
of the UES drop as well. The last force also can be injured if the peripheral nerve that innervates the UES is damaged by endotracheal intubation. The BoT injection can also be helpful for a patient in the case of a paralyzed UES caused by peripheral nerve injury. In this regard, this study will be helpful when considering the BoT injection in patients with PED who have no neurological disorders as presented.

The study of Partik et al. [4] analyzed the incidence of VFSS of 21 patients who had undergone prolonged intubation, but there was not a specific type or pattern observed by this literature review. The results of that study showed a large variety of aspiration types and associated swallowing disorders. In this case, the patient also showed multifocal abnormalities in VFSS and received swallowing training including thermos-tactile stimulation, chin tuck posture, Shaker exercise, Mendelsohn maneuver and FES with dietary modification for the first few weeks. But the symptoms did not improve. Consequently, we made a BoT injection for abnormalities as noted in the patient's UES. The UES relaxation of the patient considerably improved and the amount of pyriform sinus residue decreased after the injection was administered. It is noted that if the IL is performed only, the hoarseness and aspiration may have improved but other symptoms would not have changed in this case [8]. Therefore, this result is significant to our study, and it strongly suggests that the pathology of UES could be the main cause of PED at least in this case. If the BoT injection and the IL were separated, the effects of BoT injection could be much clearer as reviewed in this case.

The 10 units of BoT were injected bilaterally, and the total doses utilized in this case were 20 units. The determination on what dosage to use had been calculated and was therefore based on previous studies, that have reported effective use of doses between 5 and 50 units, while including up to 100 units [9]. It is noted that excessive doses can reduce UES tone not only when it is necessary but also at the status of resting, therefore it is also noted that this dose can increase the risk of food reflux from the esophagus with consequent post-deglutition aspiration into the airways [5].

This study presents several limitations, which will be explained. The laryngeal electromyography was not performed for potential recurrent laryngeal neuropathy (RLN) because the patient refused this procedure test.
Although it is usually considered a rare instance, the endotracheal intubation can cause RLN [10]. Another limitation is that there were no long-term follow-ups performed in this study. It is noted that about 2 months after the patient's BoT injection, the follow-up VFSS showed aggravation of the patient's condition. This aggravation of the patient's condition appears to be due to the reduction of the BoT injection effect, because the effect is usually sustained for about 2-6 months in most patients who have received an injection. However, further follow-up was not performed in this case, because the patient no longer visited hospital. Further study on the long-term effects of BoT injection in patients with PED is still necessary to confirm similar outcomes of this case study.

In this case, the patient received a combination of the conventional PED treatment with other treatment options, but unfortunately it was not effective to manage the patient's issues. Whereas it was similarly noted that the BoT injection for the patient made great improvement to the patient's condition. This study therefore suggests that the pathology of UES could be the main cause of the patient's incidence of PED, and further study is needed to investigate the effectiveness of the BoT injection in patients with PED.

\section{CONFLICT OF INTEREST}

No potential conflict of interest relevant to this article was reported.

\section{REFERENCES}

1. Rassameehiran S, Klomjit S, Mankongpaisarnrung C, Rakvit A. Postextubation dysphagia. Proc (Bayl Univ Med Cent) 2015;28:18-20.

2. Bishop MJ, Weymuller EA, Fink BR. Laryngeal effects of prolonged intubation. Anesth Analg 1984;63:33542.

3. Barker J, Martino R, Reichardt B, Hickey EJ, RalphEdwards A. Incidence and impact of dysphagia in patients receiving prolonged endotracheal intubation after cardiac surgery. Can J Surg 2009;52:119-24.

4. Partik B, Pokieser P, Schima W, Schober E, Stadler A, Eisenhuber E, et al. Videofluoroscopy of swallowing in symptomatic patients who have undergone long-term intubation. AJR Am J Roentgenol 2000;174:1409-12. 
5. Alfonsi E, Merlo IM, Ponzio M, Montomoli C, Tassorelli C, Biancardi C, et al. An electrophysiological approach to the diagnosis of neurogenic dysphagia: implications for botulinum toxin treatment. J Neurol Neurosurg Psychiatry 2010;81:54-60.

6. Woisard-Bassols V, Alshehri S, Simonetta-Moreau M. The effects of botulinum toxin injections into the cricopharyngeus muscle of patients with cricopharyngeus dysfunction associated with pharyngo-laryngeal weakness. Eur Arch Otorhinolaryngol 2013;270:80515.

7. Goldsmith T. Evaluation and treatment of swallowing disorders following endotracheal intubation and tracheostomy. Int Anesthesiol Clin 2000;38:219-42.

8. O'Leary MA, Grillone GA. Injection laryngoplasty. Otolaryngol Clin North Am 2006;39:43-54.

9. Kocdor P, Siegel ER, Tulunay-Ugur OE. Cricopharyngeal dysfunction: a systematic review comparing outcomes of dilatation, botulinum toxin injection, and myotomy. Laryngoscope 2016;126:135-41.

10. Spataro EA, Grindler DJ, Paniello RC. Etiology and time to presentation of unilateral vocal fold paralysis. Otolaryngol Head Neck Surg 2014;151:286-93. 